\title{
Repères
}

\section{Réflexivité et registres d'interdisciplinarité. Une boussole pour la recherche entre natures et sociétés}

\author{
Marion Borderon ${ }^{1}$, Arnaud Buchs ${ }^{2}$, Vincent Leblan ${ }^{3}$, Elisa Vecchione ${ }^{4}$ \\ 1 Géographie, chercheuse au Z_GIS, Universität Salzburg, doctorante à Aix-Marseille Université, UMR 7300 Espace, 13100 Aix- \\ en-Provence, France \\ 2 Économie institutionnaliste, maître de conférences, Université Toulouse - Jean Jaurès, UMR Dynamiques rurales, 31000 Toulouse, \\ France \\ 3 Anthropologie, chargé de recherche, IRD, UMR 208 Paloc (IRD et MNHN), 75005 Paris, France \\ 4 Politiques publiques, chargée de recherche, London School of Hygiene \& Tropical Medicine, Global Health and Development \\ Dep., WC1H 9SH, London, United Kingdom
}

Cet article est le fruit d'un travail de réflexion réalisé à l'occasion du colloque à Cerisy-la-Salle qui marquait les vingt ans de la revue NSS. Un collectif de quatre jeunes chercheurs s'interroge dans le présent texte sur les registres d'interdisciplinarité dans les processus d'évaluation. Les auteurs proposent une boussole pour le cheminement interdisciplinaire associant des enjeux dynamiques, épistémologiques et une vision réflexive particulièrement salutaire dans une période où, plus que jamais, l'évaluation de l'interdisciplinarité reste à construire. Nul doute que cette démarche et ces propositions aideront à renouveler les pratiques de la recherche et guideront les collectifs présents et à venir sur le chemin d'une interdisciplinarité assumée et ouverte à des interactions avec la société civile.

La Rédaction

Mots-clés : réflexivité ; interdisciplinarité ; objectivation ; pratiques d'évaluation

Résumé - Cet article est la réflexion commune de quatre «jeunes chercheurs » sur les pratiques d'évaluation de la recherche interdisciplinaire. Nous souhaitons comprendre comment qualifier des registres d'interdisciplinarité afin d'accroître leur transparence méthodologique. Nous proposons tout d'abord un état des lieux des modes d'objectivation de l'interdisciplinarité. Puis nous insistons sur le caractère continuellement inédit de la construction des objets interdisciplinaires, appelant la création de catégories d'évaluation dynamiques. Nous proposons enfin une boussole à cinq axes afin d'orienter l'évaluation des pratiques interdisciplinaires sur l'interface natures/sciences/sociétés: dynamique temporelle de l'interdisciplinarité, distance disciplinaire des différents corpus, dimension collective de la recherche, formes d'engagement dans le débat public et portée extra-académique.

\section{Keywords:}

reflexivity; interdisciplinary; objectivation; evaluation practices

\begin{abstract}
Reflexive patterns of interdisciplinarity. A compass for research linking nature and society. This paper opens new avenues for reflection on interdisciplinarity via the combinatory perspective that four "young researchers" propose on research evaluation practices, including editorial practices, call for proposals, teaching, recruitment, etc. For instance, all interdisciplinary journals impose specific requirements on their incoming authors. With this paper we wish to take their standpoint in order to highlight, discuss and organize these requirements into a usable product - a compass - that could eventually guide processes of interdisciplinary work evaluation. Therefore, our analysis is driven by the question of how to qualify patterns of interdisciplinarity in order to improve the methodological transparency of evaluation practices. Further, taking the example of editorial practices we start by presenting a brief inventory of the way in which interdisciplinarity gets objectified through practices,
\end{abstract}

Auteur correspondant : V. Leblan, vincent.leblan@ird.fr

L'ordre des auteurs est alphabétique. Chacun des auteurs a contribué de façon égale à l'écriture de cet article. 
from the identification of the authors' disciplinary background via their institutional affiliations to the acknowledgement of combinatory approaches via the use of bibliometric measures. We then insist on the scarcely fixable, ever-changing character of the construction of interdisciplinary objects. We take this feature as the stepping point to revisit evaluation categories in order to make them more suitable to the dynamic process of interdisciplinary construction. Our goal is not to provide an additional analysis of the interdisciplinary object itself; rather we aim to provide a tool to scrutinize the author's interdisciplinary intents in relation to his/her own creation of the research object and his/her own objectivation perspective. Therefore, we propose a compass consisting of 5 axes, each reflecting a specific aspect of interdisciplinary practices at the interface between natures/sciences/societies: temporal dynamics of interdisciplinarity, distance between disciplines, collective dimensions of research practice, style of engagement in public debate and extra-academic scope.

\section{Introduction}

En septembre 2013 à Cerisy-la-Salle, s'est tenu le colloque "Interdisciplinarités entre natures et sociétés » organisé par l'association «Natures Sciences Sociétés Dialogues » qui porte la revue éponyme ${ }^{1}$. L'enjeu était de retracer l'évolution des enjeux sociaux, épistémologiques et politiques de la production des savoirs interdisciplinaires à la jonction sociétés/environnements depuis la création de NSS, vingt ans auparavant (Arnauld de Sartre et Petit, 2013).

Hormis les autres participants et conférenciers, seize «jeunes chercheurs » ont été sélectionnés par le comité d'organisation, invités au colloque et répartis en quatre groupes de travail. Ils devaient restituer leur réflexion collective à l'occasion d'une table ronde clôturant la semaine. Si la commande de retour d'expérience, d'analyse critique des pratiques actuelles et de propositions de perspectives était relativement souple afin de laisser à chaque groupe la possibilité de se l'approprier, les regroupements ont été imposés par les organisateurs sur la base de plusieurs critères visant à favoriser le pluralisme. Chaque groupe de quatre associait des jeunes chercheuses et chercheurs qui ne se connaissaient pas auparavant, au parcours plus ou moins avancé (doctorants, post-doctorants et jeunes recrutés) et aux ancrages disciplinaires distincts. Enfin, chaque groupe était encouragé à échanger avec un interlocuteur " senior » de son choix parmi les autres participants. Le présent article restitue le travail conjoint mené par un de ces quatuors au cours du colloque et poursuivi depuis.

Le premier enjeu relevait de la nécessité de se comprendre. En effet, comment mettre «en réseau » nos quatre domaines de recherche respectifs, allant des vulnérabilités sociales face au paludisme à Dakar (Marion Borderon), à la gouvernance des ressources hydriques en Méditerranée et en Suisse (Arnaud Buchs), en passant par les rapports entre incertitude scientifique et prise

1 www.ccic-cerisy.asso.fr/interdisciplinaires13.html de décision publique concernant le changement climatique (Elisa Vecchione), ou encore les relations socioécologiques entre humains et primates en Afrique de l'Ouest (Vincent Leblan) ? La seule optique pouvant donner du sens à une telle diversité d'objets consistait à insister sur la dimension relationnelle et réflexive de nos recherches, et ce à toutes les étapes de la démarche scientifique : de la construction d'objets a priori hétérogènes aux plans académique et épistémologique, aux processus plus ou moins enchevêtrés de production de connaissances et $\mathrm{d}$ 'action publique. Un autre aspect nous confère une identité et des dispositions scientifiques communes, celui d'être issus d'une même "génération ANR ». Celle-ci se distingue par l'importance de la recherche sur contrat, avec des conséquences évidentes à tous les niveaux : formulation des problématiques, temporalités de la production et de la mise à l'épreuve des connaissances, réorganisation des rapports entre scientifiques, commanditaires et publics, sans omettre l'essor récent des pratiques de recherche collective (surtout dans les sciences sociales) et leur impact sur la formation scientifique.

Durant son parcours, chacun d'entre nous a eu à situer ses propres travaux dans un paysage institutionnel toujours fortement marqué par les identités disciplinaires, du point de vue des institutions en charge de l'évaluation des carrières jusqu'aux revues qui continuent à exiger, à tort ou à raison, que l'on se situe dans une discipline particulière (en exergue d'un article, par exemple). La conséquence de ces usages est donc souvent celle d'injonctions contradictoires entre les processus de formation interdisciplinaire dans lesquels nous nous sommes engagés et les procédures d'évaluation de la recherche. Sans être disjoints, ces deux processus restent toutefois différents. Dans cette contribution, nous nous focalisons sur le second, et plus particulièrement sur l'évaluation des pratiques interdisciplinaires. L'objectif est de qualifier des formes d'interdisciplinarité grâce à une nouvelle perspective qui met en lumière l'aspect dynamique de la constitution de l'identité d'un scientifique. Pour ce faire, nous repartons des vœux d'interdisciplinarité exprimés dans la présentation de la 


\section{Les auteurs}

Marion Borderon est chercheuse en géographie. Son parcours académique est principalement monodisciplinaire, mais les thématiques sur lesquelles elle travaille et les projets européens dans lesquels elle est impliquée l'amènent très souvent à des collaborations et des questionnements interdisciplinaires.

Arnaud Buchs est enseignant-chercheur en économie institutionnaliste. Avec pour dénominateur commun le recours au terrain dans une perspective diachronique, ses recherches portent sur l'analyse des politiques et des règles qui encadrent la régulation des ressources en eau et de leurs usages, notamment en Méditerranée et en Suisse (pénurie en eau et sécurité hydrique, gestion intégrée par bassin, etc.).

Vincent Leblan est chercheur en anthropologie. Ses travaux intègrent ethnologie, primatologie de terrain et histoire environnementale dans l'étude des relations entre hommes et primates en Afrique de l'Ouest à des fins d'anthropologie fondamentale et de recherches sur les politiques de patrimonialisation de la nature.

Elisa Vecchione est chercheuse en politiques publiques de la santé auprès de la London School of Hygiene and Tropical Medicine de Londres. Son parcours académique est marqué par des déviations disciplinaires continues débutant avec l'économie du risque. Depuis lors, elle étudie à travers des projets variés les pratiques épistémiques et normatives de l'incertitude scientifique ainsi que le lien entre les deux.

revue $N S S^{2}$ sur internet en adoptant le regard d'un comité de sélection qui se trouverait par exemple dans la posture de qualification et d'évaluation de programmes de recherche, d'articles ou encore de candidats à un poste académique. Il s'agit de proposer un outil applicable à tout processus d'évaluation de l'interdisciplinarité afin de qualifier les étapes des pratiques scientifiques en fonction de leur style d'intégration des disciplines. Leur diversité et les nombreux moyens de «faire» de l'interdisciplinarité nous ont amenés à nous interroger quant à la possibilité de les reconnaître et de les classifier. Quels critères pouvons-nous alors mobiliser afin d'objectiver les pratiques de recherche interdisciplinaire? Comment peut-on distinguer et qualifier des registres d'interdisciplinarité en dépassant la polysémie de multi-, pluri-, et transdisciplinarité, et ainsi accroître la transparence méthodologique des processus d'évaluation? Pour ce faire, nous proposons une boussole pour la recherche typiquement interdisciplinaire entre natures et sociétés, scénario qui nous a fédérés dans cette démarche réflexive à propos de la polysémie de la notion d'interdisciplinarité.

\section{Du côté des pratiques d'évaluation : état des lieux}

L'évaluation est un procédé quasi permanent dans la vie d'un chercheur actif : évaluation lors d'un processus de recrutement ou dans l'objectif d'une promotion, évaluation par son/ses organismes, évaluation de chaque article soumis, de chaque demande de financement, etc. Or, à l'instar de la recherche internationale, l'évaluation

2 Site de la revue NSS (rubrique «Présentation », onglet «Objectifs et domaines ») : http:/ / www.nss-journal.org. est un aspect peu envisagé par les institutions et les scientifiques en France (Joulian et al., 2005 ; Milard, 2010). La question spécifique de l'évaluation des actions et des pratiques interdisciplinaires ajoute donc une difficulté dans

" un monde où la recherche et les institutions se sont historiquement formées en disciplines spécialisées, où la construction des connaissances s'opère souvent dans des cadres paradigmatiques et des dispositifs extrêmement contraignants (ceux des différentes sections du Comité national du CNRS, par exemple) et générateurs d'exclusion ("ceci est hors de notre champ"), peu congruents avec l'émergence de novations intellectuelles ou de collaborations transversales » (Joulian et al., 2005, p. 285).

Dès 1970 par exemple, le CNRS (Centre national de la recherche scientifique) a mis en œuvre des actions interdisciplinaires de recherche. Cette démarche est dite cohérente avec les lignes directrices de l'organisme pour assurer la complémentarité entre disciplines voisines et la pluridisciplinarité d'objectifs. Il faut cependant attendre 2002 pour qu'une première commission interdisciplinaire voie le jour (assurant alors les carrières des chercheurs impliqués dans cette aventure). Plus récemment, en 2011, une Mission pour l'interdisciplinarité est créée au sein de l'organisme. La manière dont l'interdisciplinarité est considérée, pratiquée, qualifiée dans les différents axes de recherches de la mission n'est cependant pas clairement explicitée (CNRS, 2013). En outre, cette remarque semble pouvoir s'appliquer à d'autres processus d'évaluation. La plupart des revues nationales et internationales dans le champ de l'environnement, par exemple, publient tant des articles monodisciplinaires que des travaux empreints d'interdisciplinarité. Au niveau des procédures de sélection et d'évaluation des publications, les pratiques éditoriales ne semblent pas avoir pour habitude de distinguer les unes des autres. Or 
la question se pose quant à l'identification d'un article interdisciplinaire : comment l'interdisciplinarité se metelle en place, à quel(s) moment(s) du processus de recherche, avec quels instruments méthodologiques? On peut en effet distinguer plusieurs modèles de pratiques interdisciplinaires selon la nature de la question posée ou la nature de la collaboration ayant fondé l'interdisciplinarité. Par exemple, des phases monodisciplinaires peuvent alterner avec des phases interdisciplinaires (Mathieu, 1999).

Dans l'exemple des pratiques éditoriales, si une revue est explicitement interdisciplinaire, elle impose dès lors aux auteurs d'épouser cette démarche ou de la questionner. Cela devient un critère, une gageure. Mais comment qualifier les pratiques interdisciplinaires à travers les revues s'en réclamant? Cela demanderait de tenir compte de leur rôle dans la reconnaissance tant de l'interdisciplinarité en tant que régime(s) de scientificité, que de ses acteurs et des chercheurs interdisciplinaires qui ont besoin de reconnaissance, de promotion et de publications - qui donnent légitimité et forgent leur identité et leur CV (Joulian et al., 2005). La diversité des lieux de publications et de citations est révélatrice des choix, des engagements et de la représentativité des chercheurs engagés dans la pratique et la communication interdisciplinaire. Or les procédures sélectives et les normes éditoriales des revues restent obscures. Les efforts d'objectivation se contentent généralement $d$ 'approches bibliométriques et $n$ 'analysent pas le contenu des articles en profondeur (Prud'homme et al., 2012). L'exemple des trois formes de mesures bibliométriques utilisées pour discerner le degré d'interdisciplinarité d'un article est éloquent : l'identification des différentes disciplines des auteurs en fonction de leur adresse institutionnelle (Qiu, 1992), l'adéquation entre la discipline du ou des auteur(s) et celle(s) dominant la revue (Rinia et al., 2001) ou encore la prise en compte du nombre de sujets ou thématiques associés à un article par les bases de données Web of Science ou Scopus (Levitt et Thelwall, 2009). Enfin, l'approche la plus communément utilisée dans les revues de littérature se limite aux références et aux citations, en considérant l'hypothèse que la diversité des disciplines qui citent un article ou qui sont citées par celui-ci refléterait son degré d'interdisciplinarité.

\section{Objectivation et réflexivité}

Aussi intéressantes soient-elles, ces mesures ignorent les pratiques qui ont lieu plus en amont. Elles correspondent à une compréhension de l'interdisciplinarité qui ne dépasse pas l'assemblage de différentes disciplines audelà d'un certain renforcement mutuel en vue de la production de résultats. L'opération scientifique qui est sousjacente à l'exercice interdisciplinaire est pour ainsi dire appréhendée dans sa seule dimension de conformité à un espace d'évaluation déjà établi. Cependant, une dimension cruciale de l'opération scientifique réside dans la construction de l'objet de recherche par le chercheur. Selon Bachelard (1969, p. 239), le savant « ne peut prendre pour une pensée objective une pensée qu'il n'a pas personnellement objectivée ». Comprendre la particularité de la posture de chaque chercheur, qu'il soit disciplinaire ou interdisciplinaire, est ainsi fondamental pour comprendre le sens de son travail de synthèse. Cependant, l'évaluation des recherches interdisciplinaires est complexe du fait qu'elles ne s'imposent pas de facto, qu'elles n'émergent pas spontanément ; au contraire, elles s'argumentent par le choix d'une démarche spécifique et sont ancrées dans un contexte (Hubert et Bonnemaire, 2000). La construction des objets interdisciplinaires est ainsi continuellement inédite, nécessitant d'être dynamique dans nos catégories d'évaluation. Dès lors, il n'y a d'autre choix que de procéder à une étude du contenu de chaque article, de chaque programme, de chaque candidature, etc., pour saisir la nature de l'interdisciplinarité revendiquée (Schummer, 2004 ; Porter et al., 2008). Leur évaluation ne peut avoir un sens que si elle est menée qualitativement, c'est-à-dire, contextuellement.

À titre d'exemple, une comparaison rapide d'articles parus dans le dossier «Interdisciplinarité » de la revue $N S S^{3}$ révèle différentes façons de catégoriser et de distinguer les pratiques interdisciplinaires, ces définitions pouvant être rapportées à des motivations variées. Ainsi, les analyses ayant pour ligne de mire les enjeux institutionnels de l'interdisciplinarité, c'est-à-dire une traduction en termes de "pilotage de la recherche », la conçoivent plutôt comme une pratique de fédération de chercheurs appartenant à des disciplines différentes autour d'un thème, visant à varier les éclairages d'un même objet (Aubertin à l'IRD, 2004 ; Deffontaines et Hubert à l'Inra, 2004). La dimension collective et socialisée de la recherche interdisciplinaire est somme toute monnaie courante, et sans doute même inévitable car :

«Par définition, les étudiants sont formés via des ensembles plus ou moins disciplinaires et les chercheurs sont socialisés aux paradigmes et modes de travail de communautés spécifiques. La créativité faisant son œuvre, ces cadres de travail et de pensée se trouvent toutefois régulièrement mis en porte-à-faux - et de l'interdisciplinarité apparaît, des champs voisins se fécondent, de nouveaux cadres d'appropriation se déploient » (Pestre, 2004, p. 195).

Notons qu'un nombre croissant de jeunes chercheurs, revenant sur leurs parcours d'apprentissage, définissent aujourd'hui plus volontiers l'interdisciplinarité comme

\footnotetext{
3 Le dossier « Interdisciplinarité » de la revue NSS regroupe des articles qui participent à la caractérisation des pratiques interdisciplinaires. Les parutions sur ce thème ont été particulièrement nombreuses entre 2004 et 2006.
} 
une pratique individuelle, soulevant des enjeux épistémologiques d'autant plus prégnants qu'ils sont constamment confrontés à des instances d'évaluation disciplinaires refusant une légitimité académique à leur démarche (Bühler et al., 2006 ${ }^{4}$ ).

Cette transformation tient vraisemblablement à l'évolution des situations d'apprentissage de la recherche durant la dernière décennie : là où l'interdisciplinarité résultait surtout d'un échange entre spécialistes ayant appris à forger leurs hypothèses au sein $\mathrm{d}^{\prime}$ institutions fortement disciplinarisées, on assiste à l'arrivée de chercheurs ayant assimilé dans un même élan, via les projets ANR et la création des UMR, les concepts et les instruments de plusieurs sciences (Leblan, sous presse).

Pour l'ensemble de ces raisons, l'interdisciplinarité exacerbe la nécessité d'une démarche réflexive qui puisse mettre en relation deux éléments fondamentaux de la définition de l'identité du chercheur interdisciplinaire : la pratique scientifique au cours de sa trajectoire de recherche et son évaluation. Cette réflexivité «augmentée » résulte d'une attention particulière portée au processus de construction de l'objet de toute recherche (Hubert et Bonnemaire, 2000). À l'amont, cette attention amène à accroître la transparence des modes d'objectivation des savoirs coconstruits sur la base d'apports disciplinaires pluriels; en aval, elle contribue à renforcer la responsabilité de tout organisme ou organisation appelé à évaluer la recherche interdisciplinaire (telle qu'une revue interdisciplinaire, par exemple) vis-à-vis de la communauté scientifique et de la société en général.

Considérant que les critères d'évaluation de la pertinence des contributions interdisciplinaires résultent de choix, il importe selon nous d'aider la prise de décision grâce à des indicateurs qui permettent à la fois de dessiner une trajectoire ou politique de recherche claire et, en même temps, $d$ 'induire une introspection plus profonde de la pratique interdisciplinaire au sein de toute institution engagée à son développement. Cette nécessité n'est certes pas nouvelle. Par exemple, dans le cas de la revue NSS, son comité de rédaction avait identifié, lors d'un exercice d'autoévaluation, 4 types d'articles interdisciplinaires : 1) les articles traitant $d^{\prime} u n$ programme interdisciplinaire ; 2) ceux autour d'un thème, d'un objet de recherche, provenant d'auteurs de disciplines différentes ; 3) ceux traitant $\mathrm{d}^{\prime}$ « objets déformés » où un auteur d'une discipline écrit sur un objet de recherche pour d'autres disciplines; 4) les articles traitant de la

\footnotetext{
$4 \quad$ D'autres jeunes chercheurs jugent, pour leur part, que les pratiques interdisciplinaires dans le cadre des recherches financées par contrat affaiblissent les discussions épistémologiques sur « [...] la scientificité des sources, les protocoles de recherche, les modalités d'administration de la preuve [...]» en raison, notamment, du pouvoir des commanditaires et de l'importance accordée aux « produits finis » (Goxe et Nollet, 2006, p. 16-17).
}

question de l'interdisciplinarité en elle-même (Évaluation interne revue NSS 2005-2008).

Cependant, cette procédure d'évaluation qualifie des types d'interdisciplinarité à partir de l'objet construit et de la diversité des disciplines qu'il « rassemble» (en retraçant ses sources), tout en laissant de côté le constructeur de l'objet, c'est-à-dire la spécificité de sa démarche : selon que l'objet scientifique soit construit de façon solitaire ou collective; de manière plus «compacte » ou plus « déformée »; en utilisant des disciplines reconnues comme voisines ou s'affranchissant de ces barrières académiques et épistémologiques. Nous portons alors notre attention sur la construction de l'objet de recherche à partir de l'intention des chercheurs quant à leur trajectoire et à leur perspective d'objectivation, afin de mieux appréhender leur propre processus réflexif.

\section{Une boussole pour le cheminement interdisciplinaire}

La qualification usuelle des pratiques interdisciplinaires (mono-, multi-, inter-, trans- et pluridisciplinaire) revêt un caractère formel qui ne permet pas de rendre compte finement de la construction de l'objet de recherche. Nous proposons donc de diriger la réflexion vers l'amont de la pratique interdisciplinaire à travers une boussole dont l'objectif est moins d'aboutir à une (autre) catégorisation formelle que d'appréhender la teinte $\mathrm{d}^{\prime}$ une contribution donnée et de dessiner les contours du registre d'interdisciplinarité dans lequel elle s'inscrit (voir Fig.).

Nous proposons cinq axes, chacun renvoyant à des critères non normatifs d'appréciation de l'interdisciplinarité, visant à qualifier sa teinte à travers un type d'intégration interdisciplinaire « en actes ». Le centre de cette boussole correspond à un niveau zéro d'intégration ; audelà du centre, l'interdisciplinarité s'active en fonction de circonstances contingentes au(x) chercheur(s) impliqué(s) et se structure selon des formes d'intégration spécifiques : dynamique temporelle, niveau de «transgression » disciplinaire et niveau de collaboration (dimension collective), ces trois dimensions renvoyant strictement au caractère scientifique de l'interdisciplinarité ; rapport à l'action (formes d'engagement) et portée extra-académique, renvoyant au caractère social de sa mise en acte.

\section{Dynamique temporelle}

Le processus de recherche et sa valorisation fonctionnent souvent par étapes convenues formellement: des questionnements aux hypothèses de recherche, des procédés méthodologiques engagés aux discussions des résultats apportés et, selon les intérêts en jeu, des choix de 


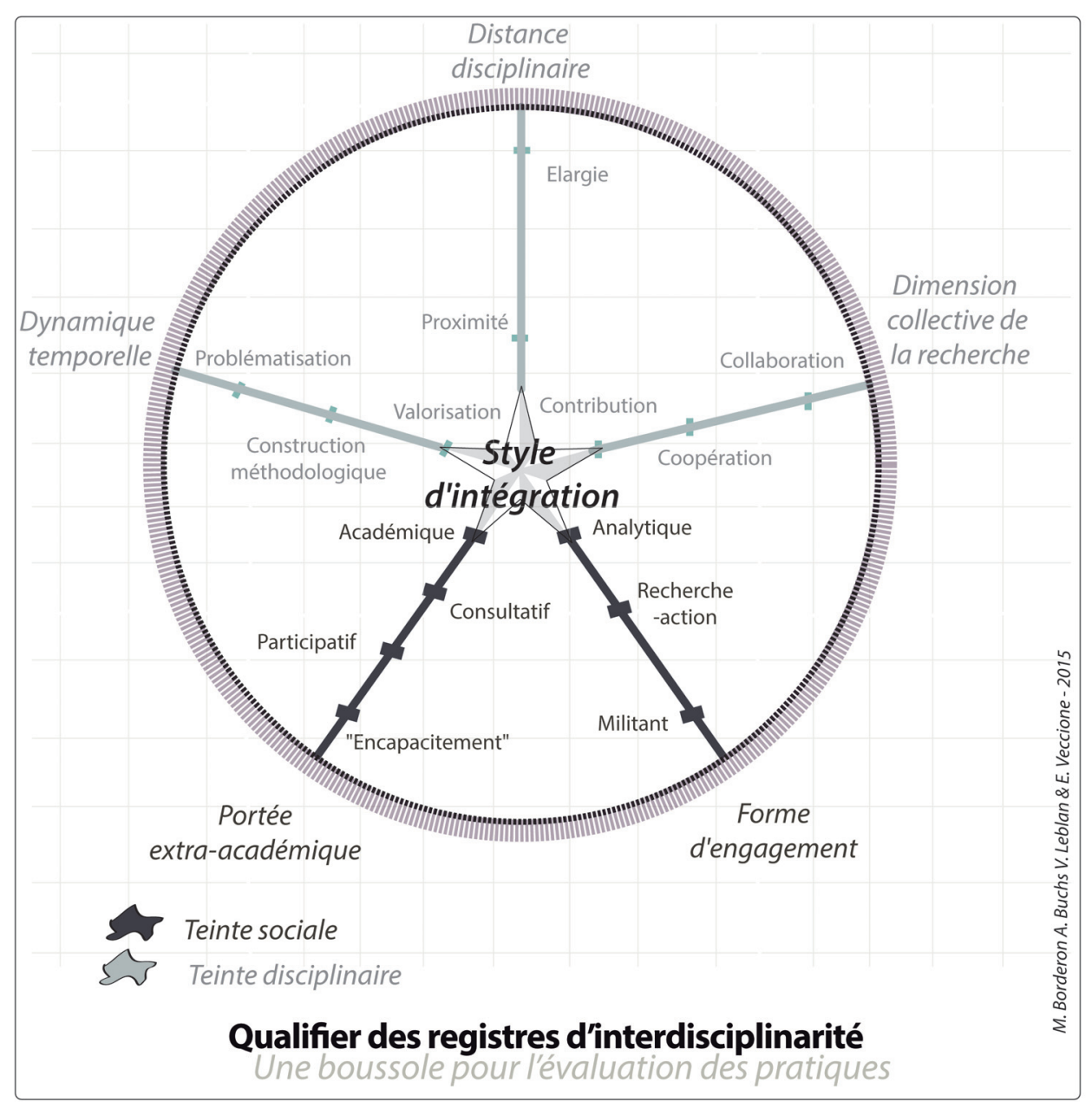

Fig. Une boussole pour qualifier des registres d'interdisciplinarité.

support pour les présenter à la communauté scientifique et les transmettre au public. Cependant, les rencontres entre disciplines ne respectent pas toujours une telle continuité ou ne s'effectuent pas toujours selon une coréflexion linéaire dans le temps. En d'autres termes, il n'est pas toujours aisé d'apprécier la dynamique temporelle de la démarche interdisciplinaire. Elle est néanmoins palpable lors de trois moments-clés de ce processus.

Juste au-dessus du degré zéro qui correspond à une recherche monodisciplinaire, l'interdisciplinarité n'apparaît qu'à l'étape d'écriture, de la diffusion et de la valorisation des résultats : c'est notamment cette conception qui semble prévaloir dans les instances de pilotage de la recherche, lorsqu'il s'agit de faire valoir un éventail de disciplines présentes au sein d'une institution ou d'un programme, sans que celles-ci ne se soient mutuellement questionnées. A contrario, une problématisation non spécifique à une discipline appellera un enracinement fort de la recherche dans une pratique d'interdisciplinarité. Dans ce cas, dès le démarrage d'un projet, l'intégration nécessite la recherche d'un compromis (sinon d'un consensus) sur le sens et la pertinence des données à observer et des méthodes à mettre en œuvre pour plusieurs disciplines mobilisées. Elle peut également conduire à la production de concepts transverses. Enfin, la situation intermédiaire caractérise une publication ou un projet dont la problématique respecte une compartimentation plutôt classique des intérêts propres à chaque discipline mais nécessitant, pour y répondre, le croisement de procédés méthodologiques issus de diverses disciplines. C'est donc seulement lors de la construction méthodologique que le(s) chercheur(s) côtoie(nt) d'autres horizons disciplinaires.

\section{Distance disciplinaire}

Une plus grande distance disciplinaire se traduit généralement par un échange plus difficile, voire laborieux. Cette situation correspond notamment à l'utilisation de méthodologies profondément différentes, telles que celles relevant des sciences sociales et des sciences naturelles. Toutefois, l'usage de concepts communs peut induire des échanges non moins laborieux. C'est le cas, par exemple, de l'opposition disciplinaire entre les 
sciences économiques et les sciences sociales, susceptible de se traduire par des « querelles de chapelles » précisément en vertu de leur proximité conceptuelle. À l'inverse, des échanges entre sciences historiques et sciences physiques, apparemment très distantes quant à l'objet de leur intérêt, peuvent être très proches par leur concept de « récits » pour la modélisation des systèmes complexes. Il s'agit donc finalement de comprendre comment s'organise la «transgression » des auteurs lorsqu'ils s'affranchissent de leur «habitat [disciplinaire] naturel» à la recherche de compromis, voire de nouveaux équilibres épistémologiques.

Ainsi, au-delà du niveau zéro, notre boussole commence à détecter une forme d'intégration horizontale entre disciplines qui, en vertu de leur « voisinage » et de leur simple juxtaposition, sont facilement appelées à se côtoyer pour finalement se renforcer les unes avec les autres. On appellera ce type d'interdisciplinarité «de proximité », en opposition à une approche dite "élargie » associant par exemple sciences de la vie et sciences de la société (Jollivet et Legay, 2005). Ce type d'approche "plus affranchie, plus ouverte» donnant lieu à des échanges plus invasifs est à ce titre qualifiée de « dédaléenne » par Bühler et al. (2006). En s'éloignant du degré zéro, on tend ainsi vers une interdisciplinarité plus fusionnelle entre disciplines grâce à une remise en cause de leurs présupposés respectifs.

\section{Dimension collective de la recherche}

Une publication ou un projet interdisciplinaire peuvent être rédigés à un ou plusieurs claviers. Il convient, le cas échéant, de clarifier les formes d'implication des auteurs dans la construction de l'exercice interdisciplinaire en question. L'ordre des signataires dans une publication, par exemple, ne constitue pas un indicateur suffisant dans la mesure où des éléments autres que la pertinence de la contribution à une recherche peuvent se jouer dans la cosignature d'un article (insertion de la publication dans une série de publications des auteurs, statuts et désirs/besoins divergents de visibilité académique, etc.). La prise en compte de cet axe doit inciter les relecteurs d'un article à en repérer la dimension collective, entendue comme le degré d'intégration intersubjective dans le processus de recherche.

Nous avons distingué trois dimensions au-delà du niveau zéro, celui-là correspondant à une recherche interdisciplinaire individuelle. Cette intégration peut relever d'une simple contribution : elle est caractérisée par un déséquilibre dans la représentation des disciplines (ainsi du sociologue-accessoire d'un programme sur l'« adaptation au changement climatique », par exemple). La coopération relève d'une démarche plus intégrative. Les auteurs sont alors d'accord : sur la pertinence d'un thème de recherche ; sur les phénomènes à observer, sans que ceux-ci ne soient nécessairement les mêmes pour chacun ; sur les objectifs visés, lesquels peuvent être connexes ou juxtaposés les uns aux autres. On peut faire l'hypothèse que ces deux premières dimensions sont celles que l'on obtient souvent en réponse à une injonction d'interdisciplinarité. Enfin, la collaboration implique un volontarisme fort pour transcender les différences disciplinaires par la recherche d'une épistémologie commune. De façon contre-intuitive, cette intégration des disciplines est plus aisée à atteindre au « niveau zéro » du collectif, c'est-à-dire lorsqu'un chercheur individuel, mobilisant plusieurs disciplines, n'a d'autres choix que « $[\ldots]$ $\mathrm{d}^{\prime}$ inventer la façon dont vont s'articuler et se nourrir les différents apports (extra-) disciplinaires qu'il mobilise » (Bühler et al., 2006).

\section{Formes d'engagement}

Ce critère vise à rendre compte $\mathrm{du}$ positionnement $\mathrm{du}$ (ou des) auteur(s) vis-à-vis de leur objet d'étude et, partant, il permet de caractériser l'enjeu de l'article le long d'un continuum positif-normatif dont les deux extrêmes seraient : d'une part, celui relevant de la pratique scientifique où les faits seraient présentés comme étant axiologiquement neutres et, d'autre part, celui relevant du dogmatisme militant. En considérant que la première borne n'est qu'illusoire et que la seconde ne relève pas de la pratique scientifique, ce continuum est restreint à trois degrés.

Le premier qualifie les articles où l'enjeu est avant tout empirique et analytique et principalement le fait d'une restitution d'observations. Le deuxième est qualifié de recherche-action: il comprend les articles dont l'argumentation conduit à l'énoncé de propositions, plus ou moins normatives, pour orienter les pratiques ou l'agenda de politiques publiques. Enfin, le troisième degré comprend les articles qui relèvent d'une forme d'engagement plus poussée, voire militante. Ce degré comprendra alors les articles dont l'objectif est de participer activement à l'agenda politique, quitte à revendiquer une certaine indépendance vis-à-vis des milieux académiques (en ancrant notamment la recherche dans les milieux associatifs, par exemple). En résumé, ce critère tend à rendre compte de l'intégration d'une démarche politique dans la pratique de recherche.

\section{Portée extra-académique}

Parmi les déterminants du style d'intégration, la mobilisation de savoirs extra-académiques doit être considérée. Il s'agit de rendre compte de l'implication directe de parties prenantes autres que scientifiques dans le processus de production de connaissances. Cela est le cas lorsqu'elles dépassent le statut « d'objet de recherche » pour devenir des contributeurs à un processus de coconstruction de savoirs et de leur diffusion (Callon, 1999). Ainsi, cet axe caractérise les dimensions 
sociales des pratiques scientifiques qui ont à cœur de sortir des frontières académiques.

Outre le degré zéro signifiant une production scientifique strictement académique, nous distinguons trois degrés d'implication des parties prenantes. Le premier correspond au recours à des processus consultatifs entre autres modalités de recueil de données empiriques (entretien et/ou questionnaire dans le cadre d'une enquête de terrain, par exemple). Le deuxième degré qualifie les démarches pour lesquelles la coproduction des savoirs via des démarches participatives est au centre de la production scientifique. Le troisième degré témoigne d'une relation plus « équilibrée » entre savoirs scientifiques et savoirs citoyens. Il correspond aux travaux qui non seulement, s'appuient sur des parties prenantes (coconstruction), mais dont l'objectif est aussi de déboucher sur la production d'outils d'aide à la décision communs. Ces compétences extra-académiques sont alors investies au cours du processus d'élaboration ainsi que pour la diffusion des connaissances, dans une perspective $\mathrm{d}^{\prime}$ « encapacitement $\mathrm{t}^{5}$ » des parties prenantes.

\section{Intérêt et application}

Appliquant la boussole à cet article, nous dirions, au plan de la «dynamique temporelle », que ce bref essai a fait l'objet d'une problématisation commune: nos accords sur la pertinence des faits à observer (l'empirie des pratiques interdisciplinaires) et sur le besoin d'un instrument d'évaluation (la boussole) tiennent sans doute à la familiarité initiale de chacun d'entre nous avec le carrefour Natures Sciences Sociétés, ainsi qu'à notre appartenance à la " génération ANR ». Pour la même raison, nous qualifierions de proximales plutôt que d'élargies nos «distances disciplinaires" réciproques. La « dimension collective » de cette réflexion relèverait de la collaboration, le contexte initial des travaux à « huis clos » $\mathrm{du}$ colloque de Cerisy-la-Salle ayant indéniablement favorisé cette dynamique. Quant à sa « forme d'engagement » et à sa " portée extra-académique », elle apparaît respectivement analytique (elle fut rédigée indépendamment de tout agenda politique, si ce n'est celui de l'évaluation scientifique) et académique (absence d'acteurs tenus par une politique publique environnementale). Rappelons cependant que la vocation de cette boussole est moins de qualifier un article isolé que de rendre comparables entre elles des pratiques scientifiques issues de processus d'interdisciplinarité différents.

\section{Conclusion}

Il est évident que ces critères ne permettent pas d'embrasser la diversité des pratiques scientifiques.

\footnotetext{
5 Les auteurs ont volontairement choisi de traduire le terme «empowerment » par ce néologisme.
}

Chaque axe comprend une myriade de nuances que seule une analyse fine peut apprécier avec précision. De plus, nous insistons sur l'idée générale que cette gradation ne permet pas de préjuger de la qualité de la démarche, seulement de la qualifier du point de vue de l'enjeu fixé par les auteurs. Comme pour le reste des critères, cette boussole ne peut suffire pour apprécier un article, un programme ou encore le profil d'un candidat interdisciplinaire. Elle ne peut donc se substituer à une analyse rigoureuse par les rapporteurs et les comités d'évaluation. Elle permet néanmoins l'adoption de critères communs pour orienter collectivement les pratiques d'évaluation interdisciplinaires. Dans une perspective réflexive, outre une contribution à l'orientation du comité, cette boussole permet également de retracer le chemin parcouru.

Loin d'être arrêtés, les critères sont ici énoncés comme des suggestions pour que chaque comité puisse adopter ceux qui lui permettent «de ne pas perdre l'orientation » quant à l'évolution de sa perspective interdisciplinaire face aux enjeux scientifiques et sociaux de la recherche entre natures et sociétés. Tout comme il ne peut y avoir une seule « bonne » interdisciplinarité, il ne peut y avoir un seul «bon thermomètre » pour en rendre compte. Néanmoins, ce qui nous semble important relève de l'adoption collégiale de ces critères au sein d'un comité et de leur remise en question périodique lors de l'examen critique. Ainsi, cette boussole ne pourra prétendre à trouver l'azimut vers un idéal, mais plutôt d'accompagner et de corriger en continu la trajectoire.

\section{Remerciements}

Les auteurs remercient les organisateurs du colloque de Cerisy-la-Salle "Interdisciplinarités entre natures et sociétés », et particulièrement Olivier Petit et Xavier Arnauld de Sartre pour la mise en place des ateliers « Jeunes chercheurs ». Nous tenons également à remercier sincèrement l'équipe du Centre culturel international de Cerisy pour son accueil.

\section{Références}

Arnauld de Sartre, X., Petit, O., 2013. NSS+20 : un collectif interdisciplinaire en mutation?, Natures Sciences Sociétés, 21, 3, 269-270.

Aubertin, C., 2004. L'interdisciplinarité victime de la réforme institutionnelle, Natures Sciences Sociétés, 12, 2, 184-185.

Bachelard, G., 1969 [1 $1^{\text {re }}$ éd.: 1938]. La formation de l'esprit scientifique, Paris, Librairie philosophique J. Vrin.

Bühler, E.A., Cavaillé, F., Gambino, M., 2006. Le jeune chercheur et l'interdisciplinarité en sciences sociales : des pratiques remises en question, Natures Sciences Sociétés, 14, 4, 392-398. 
Callon, M., 1999. The role of lay people in the production and dissemination of scientific knowledge, Science Technology $\mathcal{E}$ Society, 4, 1, 81-94.

CNRS, 2013. Mission pour l'interdisciplinarité $d u$ CNRS. Rapport d'activité 2013, Paris, CNRS-DGDS, http://www.cnrs.fr/ $\mathrm{mi} / \mathrm{IMG} / \mathrm{pdf} /$ rapport_activite_cnrs_web2.pdf.

Deffontaines, J.P., Hubert, B., 2004. Un regard sur l'interdisciplinarité à l'Inra. Point de vue de deux chercheurs du département Sciences pour l'action et le développement (SAD), Natures Sciences Sociétés, 12, 2, 186-190.

Goxe, A., Nollet, J., 2006. L'impératif interdisciplinaire des études environnementales. Eléments de réflexion sur les conditions sociales et pratiques de l'interdisciplinarité dans le cadre des recherches financées par contrat. Communication à la première journée d'étude du réseau PREVERE L'interdisciplinarité dans la recherche des SHS de l'environnement, IEP de Grenoble, 24 janvier.

Hubert, B., Bonnemaire, J., 2000. La construction des objets dans la recherche interdisciplinaire finalisée : de nouvelles exigences pour l'évaluation, Natures Sciences Sociétés, 8, 3, 5-19.

Jollivet, M., Legay, J.-M., 2005. Canevas pour une réflexion sur une interdisciplinarité entre sciences de la nature et sciences sociales, Natures Sciences Sociétés, 13, 2, 184-188.

Joulian, F., de Cheveigné, S., Le Marec, J., 2005. Évaluer les pratiques interdisciplinaires, Natures Sciences Sociétés, 13, 3, 284-290.

Leblan, V., sous presse. Les disciplines mènent à tout à condition d'en sortir. Jeunes chercheurs interdisciplinaires dans le triptyque Natures Sciences Sociétés, in Hubert, B., Mathieu, N. (Eds), Interdisciplinarités entre natures et sociétés, Bruxelles, Peter Lang.

Levitt, J., Thelwall, M., 2009. Citation levels and collaboration within library and information science, Journal of the American Society for Information Science and Technology, 60, 3, 434-442.
Mathieu, N., 1999. Interdisciplinarité interne, interdisciplinarité externe. Quel intérêt heuristique pour la géographie: réflexion à partir d'une confrontation de pratiques, in Nicolas, G. (Ed.), Géographie(s) et langage(s) : interface, représentation, interdisciplinarité. Actes du colloque IUKB-IRI de Sion, Société scientifique Ératosthène, 65-82.

Milard, B., 2010. L'évaluation de la recherche par les pairs : les risques d'une formalisation contre performante, ¿Interrogations?, 11 décembre, http://www.revue-interrogations.org/L-evaluation-de-la-recherche-par.

Pestre, D., 2004. L'évolution des champs de savoir, interdisciplinarité et noyaux durs. Communication au conseil scientifique du CNRS (14 janvier 2002), Natures Sciences Sociétés, 12, 2, 191-196.

Porter, A.L., Roessner, D.J., Heberger, A.E., 2008. How interdisciplinary is a given body of research?,Research Evaluation, 17, 4, 273-282.

Prud'homme, J., Gingras, Y., Couillard, A., Terrasson, D., 2012. Les mesures de l'interdisciplinarité. Pratiques et attitudes dans un centre de recherche français : l'IRSTEA, Note de recherche 2012-01, Montréal, Centre interuniversitaire de recherche sur la science et la technologie, UQÀM, Université de Montréal, Université de Sherbrooke, http:/ / www.cirst.uqam.ca/ Portals/0/docs/note_rech/2012-01.pdf.

Qiu, L., 1992. A study of interdisciplinary research collaboration, Research Evaluation, 2, 3, 169-175.

Rinia, E.J., van Leeuwen, Th.N., van Vuren, H.G., van Raan, A.F.J., 2001. Influence of interdisciplinarity on peer-review and bibliometric evaluations in physics research, Research Policy, 30, 3, 357-361.

Schummer, J., 2004. Multidisciplinarity, interdisciplinarity, and patterns of research collaboration in nanoscience and nanotechnology, Scientometrics, 59, 3, 425-465. 